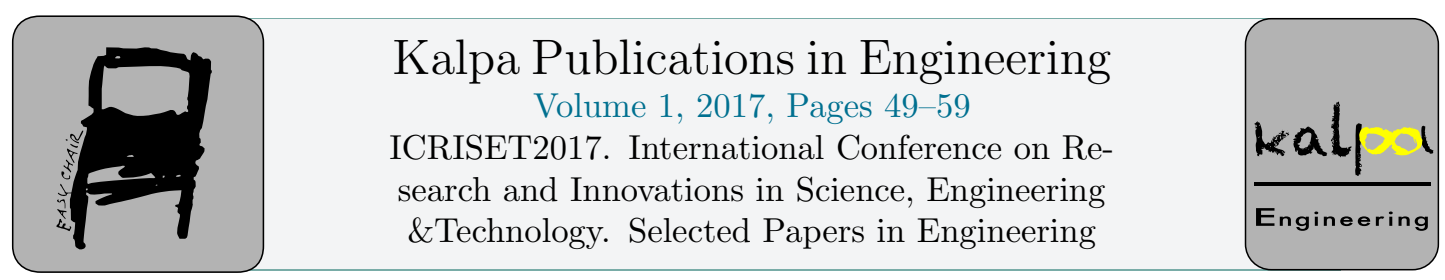

\title{
Analysis of Precoding Algorithms and Pilot Contamination Problem for Massive MIMO System
}

\author{
Omer Mohamed Abdelrhman ${ }^{1}$, Dr.Kiran R. Parmer ${ }^{2}$ and Pankaj P. Prajpati ${ }^{3}$ \\ PG Student [ECS], Dept. of ECE, L.D College of Engineering, Ahmedabad, Gujarat, India ${ }^{1}$ \\ Adjunct Professor, of ECE Aditya Physics Academy Gandhinagar, Gujarat, India ${ }^{2}$ \\ Asst. Prof., Dept. of ECE, L.D College of Engineering, Ahmedabad, Gujarat, India ${ }^{3}$ \\ $\underline{\text { Pg2017.omar@1dce.ac.in }{ }^{1} \text {, pankaj@1dce.ac.in }{ }^{2} \text { and Kparmer2006@yahoo.co.in }{ }^{3}}$
}

\begin{abstract}
Massive MIMO system has been designed to enhance the capacity, data rate, spectral efficiency and maximal energy efficiency as well as system sensitivity towards propagation environment which meets demand of future wireless communication. Using an asymptotic argument of random matrix theory, linear signal processing can be used to achieve the optimal performance of the massive MIMO. So what are the optimal numbers of antennas, actives users, and power transmitted in system? Is the one fundamental question needed to be answered? In this work we described the effect of these parameters using Ergodic achievable rate and energy efficiency by applying linear signal processing; particularly we have used zero-forcing (ZF), and maximum ratio combining or maximum transmission (MRC/MRT) algorithms. We also used minimum mean square error (MMSE) to estimate the CSI. In the context the major problem that encounters the implementation of massive MIMO multi-cell time division duplexing (TDD) network system is called pilot contamination due to limitations of coherence time. In this paper, massive MIMO along with linear precoding algorithms and pilot contamination are described and also included source and possible solutions of this problem and we found that, contrary to MRC/MRT algorithms, ZF can be used to get an optimal performance of massive when the $\mathrm{K}$ is equal to or less than BS antennas.
\end{abstract}

Keywords- Massive MIMO, pilot contamination linear precoding, channel state information (CSI), single-cell, multi-cell, Ergodic achievable rate, energy efficiency (EE).

\section{INTRODUCTION TO MASSIVE MIMO}

Massive MIMO is multi-user MIMO technology where each base station (BS) equipped with M active antennas elements and utilizes these to communicate with $\mathrm{k}$ single antenna terminals-over same time and same frequency band (Emil Björnson, 2015) also can be defined as $\frac{M}{K}>1$ constant. By coherent processing of the signals over the array antennas, downlink able to use transmit pre-coding to focus each signal to its desired terminal and receive combing can be used in uplink to discriminate between signals sent from deferent terminals (Emil Björnson, 2015). The 
more concentration beam or fine spatial beam to the terminals is achieved by using more antenna elements in the system thus more spectral and energy efficiency can be obtained. As in (YeonGeun Lim, 2015) it is shown that single-antenna user in massive MIMO system can scale down its transmit power proportionally to the number of antennas at the BS with perfect channel state information (CSI) or to the square root of the number of BS antennas with imperfect CSI (Olakunle Elijah, 2015).

Massive MIMO is one most candidate system to use in $5 \mathrm{G}$, actually there are many reasons behind one of these is ,cellular system generally deployed in urban area where many obstructions are there, thus system suffer from the propagation environment such as small scale fading and large-scale fading, fortunately, massive MIMO does not or less sensitive from the such problems. The authors in (Emil Björnson, 2015) has intensively investigated and made a comparison of the system behavior with i.i.d. Raleigh fading and line-of-sight (LOS) propagation; the authors had shown that the system gives very good performance under Raleigh fading channel. The other good advantage of massive MIMO taken from the a asymptotic arguments of random matrix theory (Yeon-Geun Lim, 2015) as number of antennas increase make possible to use simple linear signal processing approaches, such as zero-forcing ZF, matched filter MF, maximum ratio combing MRC/MRT to achieve an optimal performance of massive MIMO , and then simplicity of the system.

\section{MASSIVE MIMO SYSTEM}

This paper has analyzed massive MIMO using Ergodic sum rate to optimize the number of active users and required power transmit as well as analyzed energy efficiency for pilot contamination (numbers of pilot sequence vs number of active users). Here $\mathrm{M}$ are fixed to 128 antennas. As in fig.2 TDD frame Tc is divided into three parts first interval used as communication channel and it's called pilot signal, second interval is used for precoding signature and remain of Tc occupied by communication data. First of all we have analyzed the system under perfect CSI, then because of perfect CSI practically cannot be achieved because of cellular environment so we have also made analysis for imperfect CSI, here we have used MMSE to estimate the channel. The simulation results compared perfect CSI and imperfect CSI, power consumed by each algorithm as well as the effects of number of pilot sequences with number of active users to the energy efficiency.

\section{SYSTEM ANALYSIS AND CHANEL MODEL}

This section we first consider single cell Massive MIMO system. To choose an optimal numbers of BS antennas $\mathrm{M}$, active users $\mathrm{K}$ and transmit power $p_{t}$, we need reference to reflect the picture of $\mathrm{M}, \mathrm{K}$ and $\mathrm{p}$ effect, in linear precoding mostly they used Ergodic achievable rate for study and analysis (Yeon-Geun Lim, 2015), (Tebe Parfait, 2014), (Minn, 2015). Here we consider three Pre-coders algorithms, zero forcing ZF, maximum ratio combining or maximum transmission ratio MRC/MRT and minimum mean square ratio MMSE. Let $\mathbf{G}$ consider as linear precoding matrix $\mathbf{M} \times \mathbf{K}$ and can be written as $\mathbf{G}=\left\{\begin{array}{llllll}g_{1} & g_{2} & g_{3} & g_{k} & \ldots \ldots \ldots & g_{K}\end{array}\right\}$ and $\mathbf{H}$ is the channel matrix $\mathbf{K} \times \mathbf{M}$, where $\mathrm{H}=\left\{\begin{array}{lllll}h_{1} & h_{2} & g_{3} & h_{k} & \ldots \ldots \ldots\end{array} h_{K}\right\}, p_{t}$ is the BS transmit power. The signal received by the $\boldsymbol{k}^{\text {th }}$ user after using the linear pre-coding/ beam-forming scheme is given by as in (Yeon-Geun Lim, 2015):

$$
y_{k}=\sqrt{p_{t}} h_{k} g_{k} s_{k}+\sum_{l=1, l \neq k}^{k} \sqrt{p_{t}} h_{k} g_{l} s_{l}+n_{k}
$$


Where $\sqrt{p_{t}} h_{k} g_{k} s_{k}$ is the desired signal, $\sum_{l=1, l \neq k}^{k} \sqrt{p_{t}} h_{k} g_{l} s_{l}$ is multiuser interference and $n_{k}$ is the noise power.

From equation (1) signal-to- interference -plus-noise-ratio (SINR) of user $\mathbf{k}$ can be written as (Yeon-Geun Lim, 2015)

$$
\operatorname{SINR}_{k}=\frac{p_{t}\left|h_{k} g_{l}\right|^{2}}{\sum_{l=1, l \neq k}^{k} p_{t} h_{k} g_{l} s_{l}+1}
$$

When increasing the number of antenna elements in the analysis, the Tx power will be scaled as $1 / \mathrm{M}$ in order to compensate for the increased array gain. As a result, a constant signal-to-noise ratio (SNR) operating point is maintained $(\mathrm{Lu} \mathrm{Lu}, 2014)$.

\section{LINEAR SIGNAL PROCESSING}

By using an asymptotic argument of random matrix theory, linear signal processing can be used to achieve all of these above features. In this work we are taking zero-forcing $(\mathbf{Z F})$, and maximum ratio combing MRC/MRT I the analysis, while and minimum mean square ratio MMSE take for channel estimation.

$$
\mathbf{G}=\left\{\begin{array}{cc}
\mathrm{ZF}=H\left(H H^{H}\right)^{-1} & \text {, for zero forcing } \\
\mathrm{MRC}=H & , \text { for MRT/MTC } \\
\mathrm{MMSE}=\left(H p H^{H}+\sigma^{2} I_{M}\right)^{-1} H & , \text { for MMSE }
\end{array}\right.
$$

\section{CHANNEL ESTIMATION}

In practice, full CSI may not be directly available due to pilot contamination and feedback delay, In order to get finite CSI, we use MMSE channel estimation. The channel matrix can be estimated as in (Mingfeng HeLiang Yang, 2015.):

$$
H^{*}=\zeta \mathrm{H}+\sqrt{1-\zeta^{2}} E
$$

Where $\zeta$ is the reliability of the estimation, and $\mathbf{E} \sim \mathcal{C} \mathcal{N}(0,1)$ represents the error matrix

$$
\mathrm{V}= \begin{cases}\mathrm{F}=H^{*} & , \text { for MRT/MTC } \\ F=H^{*}\left(H^{*} H^{* H}\right)^{-1} & , \text { for zero-forcing }\end{cases}
$$

Where $H^{*}$ represents the complex conjugate transpose of the estimated channel matrix. Generally, we used to set $\mathbf{V}=\mathbf{G}$, since it reduces the computational complexity. 


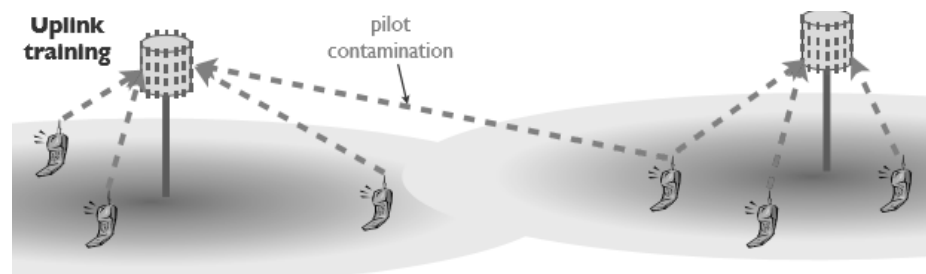

Figure 1. Pilot contamination problem

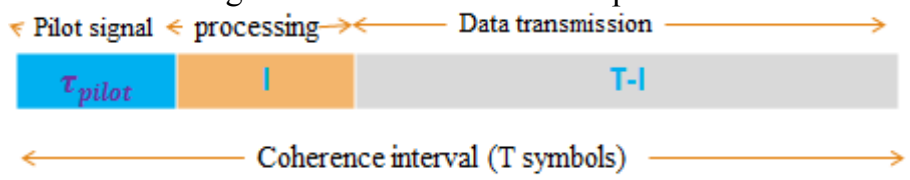

Figure.2 TDD frame

\section{LIMITATIONS OF TDD MASSIVE MIMO}

In this section, we define the major problems of the TDD massive MIMO.

\subsection{Pilot contamination}

In a multi-cell scenario as in fig.1, non-orthogonal pilots across neighboring cells are utilized, as orthogonal pilots would need a length of least $\mathrm{K} \times \mathrm{L}$ symbols $(\mathrm{K}=$ total number of Uses in a cell and $\mathrm{L}=\mathrm{a}$ total number of cell in the system) owing to frequency reuse factor of one. The use of $\mathrm{K} \times \mathrm{L}$ symbols training sequence is not feasible in practice for multi-cell as a result of short channel coherence times due to the mobility of UTs. This causes a phenomenon known as pilot contamination and it has been considered as a major impairment in the performance of massive MIMO systems (Olakunle Elijah, 2015).

\subsection{Channel reciprocity}

Environment channel communication is continually change, so that this change affect the reciprocity of TDD protocol although in (Olakunle Elijah, 2015) described that the effect of Raleigh fading is reduced in massive MIMO, also the hardware chain in the base station many not work properly because of non-linear amplification, phase noise, quantization error (Olakunle Elijah, 2015), it means that uplink and downlink lose the reciprocal property. Under this condition, the performance of the system gets more degrades.

\subsection{Sources of pilot contamination}

\subsubsection{Non-orthogonal pilot signals:}

As in fig.2, in multi-cell communication scenario the channel coherence time interval is very limit to accommodate large number of users (in full pilot reuse factor 1), there are two or more than two users in adjacent cells using same time slot this known as non-orthogonal pilot sequence, which lead to pilot contamination, therefore more increasing in intera-cell interference then degrade the performance of the system (Emil Björnson, 2015). 


\subsubsection{Error in CSI estimation}

One other source of pilot contamination is imperfection or error in CSI estimation, any error in channel state information estimation lead to pilot contamination

\section{MITIGATION METHODS OF PILOT CONTAMINATION}

In this section, we reviewed the existing methods used to eliminate or reduce the impact of pilot contamination in multi-cell massive MIMO TDD system considering full pilot reuse factor 1 .

\subsection{Time-shifted protocol}

This protocol reviewed in (Olakunle Elijah, 2015), which used to reduce the pilot contamination effect in multi-cell system, basically in this protocol scheme, transmission of pilot signals in the different cells of the system is accomplished by shifting the pilot positions in the frame such that users in different cells transmit at non-overlapping times as shown fig. 3

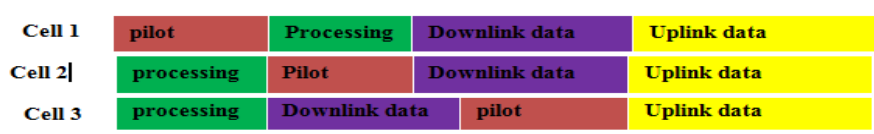

Figure 3: Time-shifted pilot scheme adapted

Eliminating of pilot contamination can be done using the proposed scheme, as long as the pilot does not overlap in time and applied appropriate power allocation algorithm. In practice may be difficult to implement this scheme, because It requires second order statistics of all the UL channels (Olakunle Elijah, 2015).

\subsection{Subspace-Based Estimation Approach}

Here Subspace estimation technique is applied using eigenvalue decomposition (EVD), the method is susceptible to error due to assumed channel between users and the BS are paired, they are orthogonally when the number of BS antennas M tends to be infinite. But in practical scenario the number of antennas finite, so in order to reduce these errors, the EVD algorithm is combined with iterative least squares and projection algorithm, in (Olakunle Elijah, 2015) results obtained described that the EVD method is not affected by pilot contamination and performs better than conventional pilot-based techniques but its accuracy depends on large number of BS antennas and increased sampling data within the coherence time.

\subsection{Blind Technique}

Blind techniques are based on subspace partitioning. Blind pilots purify technology for systems with power control switching handoff policies, and this technique can separate subspaces interference from the desired signal subspace, which can remove pilot contamination phenomenon. There is also precoding technique used for massive MIMO when systems having a limited number of antennas. 


\section{PERFORMANCE ANALYSIS}

In this section we are going to drive expression for the system performance. Which include erogdic sum rate, required transmitted power as we as energy efficiency.

\subsection{EROGDIC SUM RATE}

Ergodic sum rate can be used to describe the effectiveness of a massive MIMO system. For simply analyzing the ergodic sum rate of the system, we assume that the total downlink power is fixed and equally shared among all the users in a single cell. The sum rate can be written as :

$$
R_{k}=\log _{2}\left(1+\operatorname{SIN} R_{k}\right) .
$$

Then for $\mathrm{K}$ number of users, the ergodic sum rate is given as:

$$
R_{\text {sum }}=\sum_{k=1}^{K} E\left\{R_{k}\right\}
$$

Using (3) and substitute on (4)we shall get

$$
\begin{gathered}
R_{\text {sum }}=\sum_{k=1}^{K} \log _{2}\left(1+\operatorname{SINR}_{k}\right) \\
R_{\text {sum }}=\mathrm{K}_{\log }\left(1+\operatorname{SINR}_{k}\right) \ldots \ldots \ldots \ldots
\end{gathered}
$$

For perfect CSI system Combining (2) (7), the ergodic sum rate with MRT matrix normalization and ZF vector normalization can be respectively expressed as in (Yeon-Geun Lim, 2015)

$$
\begin{gathered}
R_{\text {sum }}^{Z F}=\mathrm{K} \log _{2}\left(1+\frac{p_{t}(M-K+1)}{K}\right) \ldots \\
R_{\text {sum }}^{M R T / M R C} \mathrm{~K} \log _{2}\left(1+\frac{p_{t}(M+1)}{p_{t}(M-1)+K}\right)
\end{gathered}
$$

For imperfect channels, there sum rate can be written as in (Mingfeng HeLiang Yang, 2015.)

$$
\begin{aligned}
& R_{\text {sum }}^{Z F}=\mathrm{K} \log _{2}\left(1+\frac{\zeta^{2} p_{t}(M-K+1)}{\left(1-\zeta^{2}\right) p_{t}+1}\right) \ldots \\
& R_{\text {sum }}^{M R T / M R C} \mathrm{~K} \log _{2}\left(1+\frac{p_{t}(M+1)}{p_{t}(M-1)+K}\right)
\end{aligned}
$$

\subsection{REQUIRED DOWNLINK TRANSMIT POWER}

In this section, we derive the total downlink transmit power required by ZF and MRT to achieve the same data sum rate according to the system under consideration. For driving transmitted power take equations (8) and (9) for perfect CSI. By considering the fact that the targeted data rate is the same for both, precoding schemes we can write

$$
\ln 2 . R_{\text {sum }}^{Z F}=\mathrm{K} \ln \left(1+P_{D}^{Z F}\left(\frac{(M-K+1)}{K}\right)\right) .
$$


Taking exponential of both sides we have

$$
P_{D}^{Z F}=\mathrm{K}\left[\frac{e^{\frac{\ln 2 R_{S \text { SI }}^{Z F}}{\mathrm{~K}}-1}}{(M-K+1)}\right]
$$

And the power transmit for MTC/MRT can be achieved using same procedure

$$
\ln 2 \cdot R_{\text {sum }}^{M R T / M R C}=\mathrm{K} \ln \left(1+P_{D}^{M R T / M R C}\left(\frac{(M+1)}{(M-1)+K}\right)\right)
$$

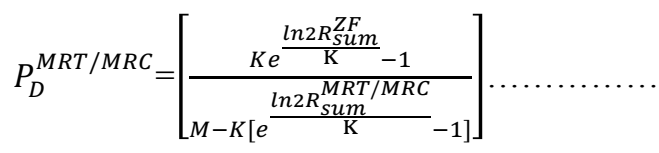

In the study and analysis of pilot contamination we applied in multi-cell scenarios under imperfect CSI. To illustrate this we consider completely symmetric scenario where the system parameters M, K, Rsum are the same in all cells and optimized jointly. The symmetric implies that the cell shape, users distribution, propagation condition are the same in all cells. Also we considered only interference that arrives from the cell neighbors. The necessity of reusing pilot resources across cells causes pilot contamination. To investigate its impact on the energy efficiency EE we consider deferent pilot reuse factor in our study. In this work we take only ZF algorithm and focus on comparing different reuse factor. An energy efficiency optimal multi-user MIMO setup is achieved in uplink and downlink by using (Emil Bjornson, 2015):

$$
\text { maximize } \quad E E=\frac{\sum_{k}^{K}\left(E\left\{R_{K}^{U L}\right\}+E\left\{R_{K}^{D L}\right\}\right)}{P_{T X}^{U L}+P_{T X}^{D L}+P_{C P}(M, K, \tilde{R})} .
$$

Where $P_{T X}^{U L}$ and $P_{T X}^{D L}$ are the power consumption required for uplink and downlink respectively, $P_{C P}$ is power consumption required for site-cooling control signaling, backhaul infrastructure and broadband processors (Emil Bjornson, 2015).

\section{SIMULATIN AND RESULTS}

Fig. 4 and fig. 5 demonstrate the achievable sum rate in numerous numbers of active users in both perfect CSI and an imperfect CSI cases with the number of BS antennas fixed to 128 antennas, estimation error $\xi$ approximated to 0.5 and also the BS downlink transmits power adequate to 0 decibel. The simulation results show that, perfect CSI case as in fig. 4 achieves higher achievable sum rate compare to imperfect CSI case as in fig.5, for both MRT and ZF. However, as the number of users increase, the achievable total rate for MRT will increase typically, however the achievable sum rate for ZF will increase first, when K reach to critical 
point, then sum rate get decreases. It implies that there's best range of users for the highest achievable sum rate once ZF is employed. The critical point in perfect CSI case is delineated as $\mathrm{pt}(\mathrm{M}+1) /(\mathrm{pt}+1)$. Moreover, imperfect CSI makes an optimal number of users and its corresponding achievable sum rate smaller compare to perfect CSI.

For designing an optimal power required consumed by the BS there are three cases: firstly, when the number of active users $\mathrm{K}$ equal to our predesigned sum rate shared by the users, there the power consumed by the two algorithms shall be same $P_{\text {sum }}^{M R T / M R C}=P_{\text {sum }}^{Z F}$ as in fig.6. As M increase through the system $\mathrm{M} \gg \mathrm{K}$, a similar total downlink transmit power is required with ZF and MRT to achieve 1 bit per second per Hertz for each user as in figure 6 .

Secondly, if we wish to attain quite more than 1 bit per second per Hertz for every user where $\mathrm{K}$ is less than predesigned sum rate, Rsum $>\mathrm{K}$ which provides $P_{\text {sum }}^{M R T / M R C}>P_{\text {sum }}^{Z F}$ as in figure 8 . Based on this analysis, we could conclude that, for the total achievable data rate equally shared among the users, as the number of transmit antennas increases with $\mathrm{M}>>\mathrm{K}$, MRT requires more power than ZF to achieve more than 1 bit per second per Hertz for each user. It implies that ZF is more power efficient to attain higher data rate.

Third, contrary to the second case if we would like to attain less than 1 bit per second per Hertz for every user there Rsum $<\mathrm{K}$ which provides $P_{\text {sum }}^{M R T / M R C}<P_{\text {sum }}^{Z F}$. Based on this analysis, we conclude that, for the total achievable data rate equally shared among the users, as the number of transmit antennas increases with $\mathrm{M}>>\mathrm{K}, \mathrm{ZF}$ requires more power than MRT/MRC to achieve less than 1 bit per second per Hertz for each user. It means that in this case MRT/MRC is more power efficient to achieve higher data rate compare to ZF as in figure.7.

In the last part of this work we were trying to design precoder for pilot decontamination and analysis of the pilot contamination problem for multi-cell scenario with imperfect CSI. We have considered pilot contamination as a special noun that represents the interference resulting in nonorthogonal pilot information in TDD which reduce the EE performance, also we have taken in a count intera-cell interference in the our multi-cell system. we have used equation (55) from (Emil Bjornson, 2015) to simulate $\mathrm{EE}$ under different pilot factors where $(\mathrm{tc}=\mathrm{Bc} * \mathrm{Tc})$, tc coherence interval, $\mathrm{Tc}$ is the coherence time, $\mathrm{Bc}$ is Bandwidth so for example coherence interval tc $=200$ symbols can be obtained with $\mathrm{Bc}=200 \mathrm{KHz}$ and $\mathrm{Tc}=1 \mathrm{~ms}$ which supports high user velocities in urban environment at $2 \mathrm{GHz}$ carrier frequencies then pilot reuse factor can be calculated as $\mathrm{f}=\mathrm{tc} / \mathrm{K}$ , in the context, when pilot reuse factor of 1 is used compared to reuse factors of 2 and 4 . The highest pilot reuse pattern $(f=4)$ gives the highest EE and so high throughput as in fig.9. The number of active users can be optimized for each $\mathrm{M}$ antenna to get good performance of the EE. This emphasizes the need to actively mitigate pilot contamination in multi-cell systems. 

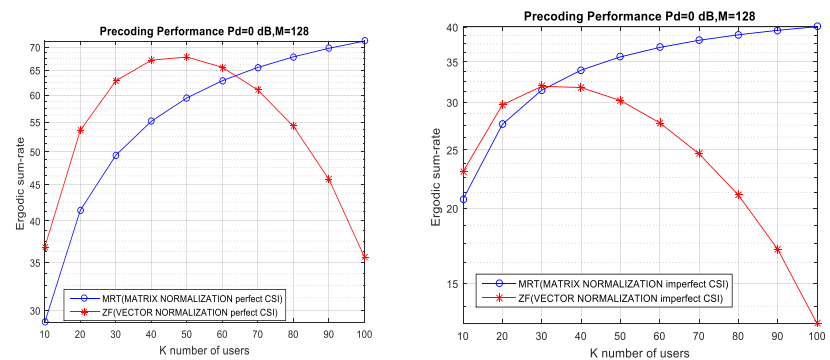

Fig.4 Ergodic sum rate versus the number of number of users $\mathrm{K}$ with perfect CSI , $\mathrm{M}=100$ $\mathrm{M}=100, p_{t}=0 \mathrm{~dB}$

Fig.5 Ergodic sum rate versus the $\mathrm{K}$ users $p_{t}=0 \mathrm{~dB}$ with imperfect CSI at and $\xi 2=0.5$

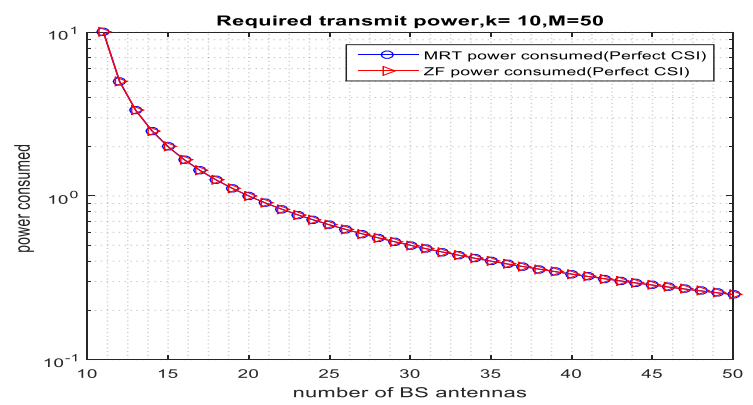

Figure. 6:Performance comparison of total downlink transmit power required to achieve more than 1 bit per second per Hertz for each user, with the number of users $\mathrm{K}=10$ and the targeted sum rate $\mathrm{Rsum}=10 \mathrm{bits} / \mathrm{s} / \mathrm{Hz}$.

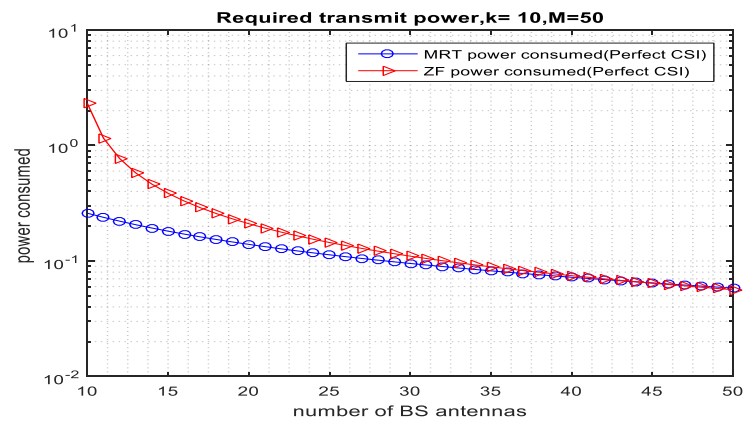

Figure 7: Performance comparison of total downlink transmit power required to achieve more than 1 bit per second per Hertz for each user, with the number of users $K=10$ and the targeted sum rate $\mathrm{Rsum}=5 \mathrm{bits} / \mathrm{s} / \mathrm{Hz}$ 


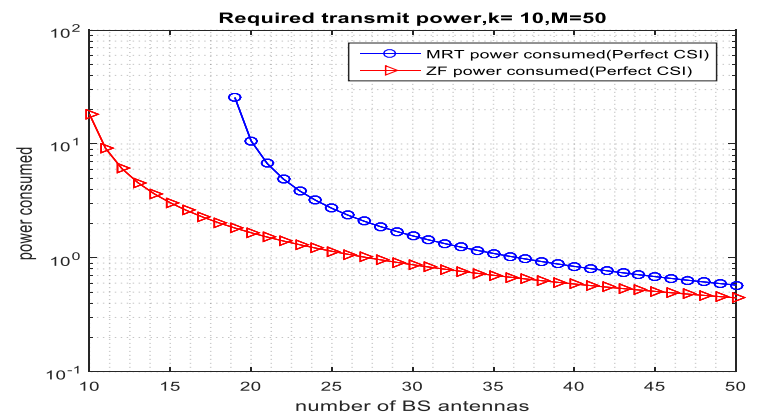

Figure 8: Performance comparison of total downlink transmit power required to achieve more than 1 bit per second per Hertz for each user, with the number of users $\mathrm{K}=10$ and the targeted sum rate $\mathrm{Rsum}=15 \mathrm{bits} / \mathrm{s} / \mathrm{Hz}$

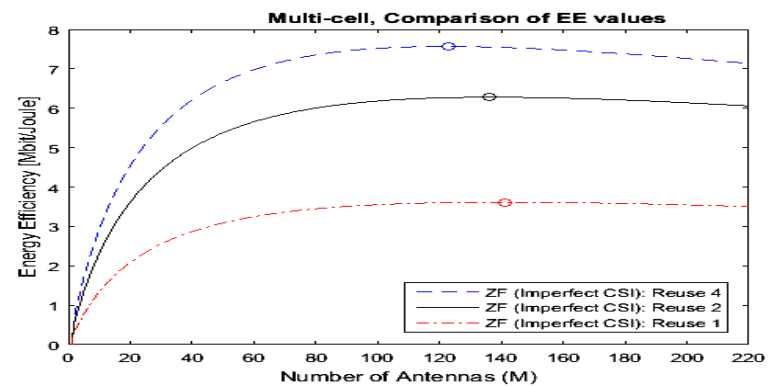

Figure 9: multi-cell EE performance using imperfect CSI, ZF with different pilot reuse factor.

\section{CONCLUSIONS}

This paper analyzed how to optimize the number of BS antennas M, number of active users $\mathrm{K}$, to get optimal sum rate (per user) which can maximized the energy efficiency EE in multi-user MIMO systems. In contrast we used realistic Erogdic sum rate model and as well as power consumption model to analysis the system. As in figs.4- 5 we found that neither MRC/MRT nor ZF achieves higher Ergodic sum rate in all the cases. In massive MIMO, contrary to the common belief, the transmit power should increase by increasing the number of antennas $M$ (due to increasing circuit components) therefore system cannot operate in low SNR regime but as appear in our simulation the power consumed per antenna is however decreasing by increasing $\mathrm{M}$, this prove that massive MIMO can be built to work on low power consumption profile depend to our parameters design (as designed data rate among all users) and algorithm that we used. To obtain high data rate ( bit per second per Hertz) ZF algorithm is preferred when $\mathrm{K}$ is less than or equal to half of $\mathrm{M}$. In the analyzing of pilot contamination issues through the system we considered only ZF imperfect CSI processing and simulated under using different pilot recuse factor, using largest reuse gives highest $\mathrm{EE}$ and that can be used to mitigate the pilot contamination problem. By optimized all terms that we described above the It would be possible to use an evolutionary approach to designing an optimal network which has all massive MIMO features. 


\section{BIBLIOGRAPHY}

Emil Björnson, E. G. (2015, Aug. 18). Massine MIMO: Ten myth and one critical Question. IEEE.

Emil Bjornson, L. S. (2015, March). Optimal Design of Energy-Efficient Multi-User MIMO Systems: Is Massive MIMO the Answer. publication in a future issue IEEE Journal.

Lu Lu, G. Y. (2014, Oct.). AnOverview of Massive MIMO: Benefits and Challenges. IEEE.

Mingfeng HeLiang Yang, H. D. (2015., December.). Performance analysis of Massive MIMO Dawnlink system with imperfect channel state information. International Journal of Research in Engineering and Science (IJRES).

Minn, A. K. (2015). Achievable Downlink Rates of MRC and ZF Precoders in Massive MIMO with Uplink and Downlink Pilot Contamination. EEE Transactions on Communications., 10.1109/TCOMM.2015.2482965.

Olakunle Elijah, C. Y. (2015, July ). Mitigating Pilot Contamination in Massive MIMO System 5G: An Overview. IEEE.

Tebe Parfait, Y. K. (2014, July). Performance analysis and comparison of ZF and MRT based downlink massive MIMO systems. IEEE.

Yeon-Geun Lim, C.-B. C. (2015). Performance Analysis of Massive MIMO for Cell Boundary Users. IEEE Transactions on Wireless Communications. 